\title{
Selectivity for Complex Shapes in Primate Visual Area V2
}

\author{
Jay Hegdé and David C. Van Essen \\ Department of Anatomy and Neurobiology, Washington University School of Medicine, St. Louis, Missouri 63110
}

To explore the role of visual area V2 in shape analysis, we studied the responses of neurons in area V2 of the alert macaque using a set of 128 grating and geometric line stimuli that varied in their shape characteristics and geometric complexity. Simple stimuli included oriented bars and sinusoidal gratings; complex stimuli included angles, arcs, circles, and intersecting lines, plus hyperbolic and polar gratings. We found that most V2 cells responded well to at least some of the complex stimuli, and in many $\mathrm{V} 2$ cells the most effective complex stimulus elicited a significantly larger response than the most effective

The neural mechanisms by which the visual cortex analyzes low-level spatial dimensions, such as orientation and spatial frequency, have been studied intensively (DeValois and DeValois, 1988; Reid and Alonso, 1996). In addition, selectivity for a variety of more complex shape characteristics has been reported in early and intermediate areas of the visual cortical hierarchy. This includes selectivity for stimulus curvature in cat area 17 (Dobbins et al., 1987; Versavel et al., 1990) and in macaque area V4 (Pasupathy and Connor, 1999), selectivity for corner-like combinations of angles in cat areas 17 (Versavel et al., 1990) and 19 (Hubel and Wiesel, 1965), and selectivity for hyperbolic and polar grating patterns in macaque area V4 (Gallant et al., 1996). In area $\mathrm{V} 2$, the focus of the present study, many cells are responsive to subjective contour stimuli (Peterhans and von der Heydt, 1993). On the other hand, Kobatake and Tanaka (1994) tested cells in the anesthetized macaque with a collection of complex stimuli and reported an absence of complex shape selectivity in V2 that was present in V4 and the inferotemporal cortex.

Altogether, these studies provide an intriguing but fragmentary understanding of how form information is processed at early and intermediate stages of cortical visual processing. One approach to enhancing our understanding is to test cells with a broader repertoire of stimulus features and dimensions than has been used previously. In the present study, we have systematically tested neurons in area V2 with a large set of visual stimuli, including simple shapes (bars and sinusoidal gratings) plus a variety of representative higher order shapes that are based on contours (e.g., angles and curves) or grating patterns. Our results indicate that area V2 is involved in analyzing a more extensive set of shape characteristics than has been previously recognized.

Received Sept. 27, 1999; revised Dec. 22, 1999; accepted Dec. 23, 1999.

This work was supported by National Institutes of Health Grant EY02091 to D.C.V.E. We thank Drs. Jack Gallant and Steven Petersen for helpful suggestions and comments and Michael Ty for valuable help during recording sessions. Many colleagues made useful comments on this manuscript.

Correspondence should be addressed to Dr. David C. Van Essen, Department of Anatomy and Neurobiology, Washington University School of Medicine, Box 8108, St. Louis, MO 63110. E-mail: vanessen@v1.wustl.edu.

Copyright (C) 2000 Society for Neuroscience $0270-6474 / 00 / 200001-\bullet \$ 15.00 / 0$ bar or sinusoid. Approximately one-third of the V2 cells showed significant differential responsiveness to various complex shape characteristics, and many were also selective for the orientation, size, and/or spatial frequency of the preferred shape. These results indicate that V2 cells explicitly represent complex shape information and suggest specific types of higher order visual information that V2 cells extract from visual scenes.

Key words: area 18; boundary; curvature; hyperbolic; nonCartesian gratings; polar; shape selectivity; surface; texture

Preliminary results of this study have been reported previously in abstract form (Hegdé and Van Essen, 1997a,b).

\section{MATERIALS AND METHODS}

Recording procedures. We recorded the responses of single units from area V2 in four hemispheres of three awake, fixating macaque monkeys using standard procedures (Connor et al., 1997). The stimulus set consisted of 48 grating stimuli and 80 contour stimuli (see Fig. 1). For the various analyses performed in this study, the stimuli were grouped into equal-sized subclasses that (with one exception) shared common shape characteristics but varied in orientation, size, and/or spatial frequency. Grating stimuli were subdivided into four subclasses (with 12 stimuli each) that shared common shape characteristics but varied in orientation and/or spatial frequency: (1) sinusoidal gratings, (2) hyperbolic gratings, (3) concentric-like polar gratings, and (4) radial-like polar gratings. For the concentric-like gratings, the concentric frequency exceeded the radial frequency, and for the radial-like gratings, the radial frequency exceeded the concentric frequency. Of the four polar gratings in which the concentric frequency and the radial frequency were equal, the pair with the two highest frequencies was assigned to the concentric-like grating subclass, and the remaining pair was assigned to the radial-like grating subclass.

The contour stimuli were grouped into 10 subclasses, each containing eight stimuli varying in orientation and size (and also in shape in the case of subclass 4): (1) bars, (2) three-way intersections, (3) crosses, (4) fiveand six-armed stars plus circles, (5) acute angles, (6) right angles, (7) obtuse angles, (8) quarter arcs, (9) semicircles, and (10) three-quarter arcs. Each contour shape was presented in two sizes, the larger matching the cell's preferred bar length and the smaller ones at half that size. Collectively, these stimuli allowed us to probe the selectivity of V2 cells for low-level form cues (i.e., orientation and spatial frequency) plus a

This article is published in The Journal of Neuroscience, Rapid Communications Section, which publishes brief, peerreviewed papers online, not in print. Rapid Communications are posted online approximately one month earlier than they would appear if printed. They are listed in the Table of Contents of the next open issue of JNeurosci. Cite this article as: JNeurosci, 2000, 0:RC61 (1-6). The publication date is the date of posting online at www.jneurosci.org.

http://www.jneurosci.org/cgi/content/full/3976 
variety of complex shape and textural characteristics. We will refer to sinusoidal gratings and bars as simple gratings and simple contours, respectively, and to non-Cartesian gratings and nonlinear contours as complex gratings and complex contours, respectively.

The cell's preferred bar parameters, including preferred length, width, color, and orientation, were determined qualitatively during the initial receptive field mapping. The stimulus set was reoriented for each cell according to the cell's preferred orientation (also see legend to Fig. 1). All stimuli were presented in the cell's preferred color (selected from a palette of six colors) over a uniform gray background. The line width of contour stimuli was determined by the cell's preferred bar width. The grating stimuli had a spatial frequency of two, four, or six cycles per receptive field diameter and had the same diameter as the receptive field and the same mean luminance as the background.

We isolated single units using standard procedures and identified units as belonging to area V2 based on visual topography and receptive field size (Burkhalter and Van Essen, 1986). Receptive field eccentricities ranged from 2.8 to $9.7^{\circ}$ (mean, $4.6^{\circ} ; n=194$ ). Receptive field diameters ranged from 1 to $3.4^{\circ}$ (mean, $1.4^{\circ}$ ). We did not attempt to histologically characterize the recording locations. Stimuli were presented within the classical receptive field sequentially for $300 \mathrm{msec}$ each with a $300 \mathrm{msec}$ interstimulus interval while the animal fixated within a window of $0.5^{\circ}$ radius for a liquid reward. Up to six stimuli per trial were presented in this manner. To reduce the contributions of any receptive field nonuniformities, each stimulus was presented at three different jitter positions spaced evenly from each other and offset from the receptive field center by $25 \%$ of the receptive field radius.

Data analysis. The time window for the analysis of visually evoked response was adjusted for each cell based on the latency and duration of the response estimated from the average peristimulus time histogram. The background response was calculated using the portion of the interstimulus interval after the off response had ceased. The response to each stimulus was averaged from the above-background firing rate during 12 repetitions of the stimulus, 4 at each jitter position ( 9 repetitions, 3 at each jitter position, for 62 cells).

Most of the statistical analyses were carried out using S-Plus software (Statsci, Seattle, WA). In cases involving multiple comparisons, we adopted a stringent approach of using the Bonferoni correction $(\alpha=$ $0.05 / n$, where $\alpha$ is the probability of Type I error and $n$ is the number of comparisons; Huberty and Morris, 1989). Randomization analyses (Manly, 1991) were used to test whether the distribution of cells in different categories was random. For the analysis of cell distributions, the test statistic was the variance of the distribution of the cells across the given set of stimulus subclasses. This test statistic was calculated for the actual population distribution and for the same number of cells assigned randomly to each of the stimulus subclasses. For the analysis of response variance, the test statistic was the variance of the cell's responses to its most effective stimuli from the given set of stimulus subclasses, calculated using the spike counts during each presentation of each of the effective stimuli. This test statistic was calculated for the actual distribution of spike counts across the subclasses and for the same set of spike counts assigned randomly to each of the subclasses. In all cases, the randomization process was repeated $10^{6}$ times. The fraction of times the variance of the random distribution exceeded that of the actual distribution represents the one-tailed probability $p$ that the actual distribution was random (Manly, 1991).

Each cell included in the study had at least one stimulus for which the evoked response differed from the background response at a significance level of $p<0.05$ (two-tailed $t$ test with Bonferoni correction). Of the 196 cells recorded from in V2, 180 cells passed this test and were included in this study.

\section{RESULTS}

\section{Selectivity of V2 cells for complex contours and gratings}

Two exemplar cells, each of which showed a clear preference for complex over simple geometric stimuli, are shown in Figure 1 using a color-coded response display. The cell illustrated in Figure $1 A$ was maximally driven by a right-angle contour stimulus. This cell was very narrowly tuned, with only two other stimuli (an acute angle and a semicircle) eliciting greater than a halfmaximal response. The response to the best right-angle stimulus $[63 \pm 4(\mathrm{SEM})$ spikes/sec] was not predictable from the responses to simple bar stimuli, because the cell responded poorly (17 spikes/sec) to bars presented at one of the component orientations and not at all at the other component orientation. The cell's shape selectivity is particularly noteworthy, because little or no response was elicited by many other stimuli that contained both of the component orientations of the preferred right angle (i.e., other right-angle stimuli, intersections, and hyperbolic gratings). The greater effectiveness of angle and arc stimuli in the third row of Figure $1 A$ compared with those in the first row signifies a selectivity for the polarity of the angle and/or the sign of curvature, not simply a deviation from colinearity. In other words, the cell was selective not only for the geometric shape per se but also for the orientation of the most effective shapes. Importantly, for this cell (and for the cells in our sample in general), the response magnitude was reasonably consistent for stimuli presented at each of the three jitter positions within the receptive field (see Materials and Methods). This indicates that stimulus selectivity was not attributable to nonuniformities within the classical receptive field or to trial-to-trial fluctuations of eye position within the fixation window (data not shown).

Figure $1 B$ shows another cell that was highly selective for complex contour stimuli, including arcs (especially three-quarter arcs of all orientations) and a circle, but only for the larger of each stimulus pair. In contrast to the preceding example, this cell responded well to a broad spectrum of grating stimuli. The most effective grating stimuli included concentric rings and high frequency spirals, which is consistent with the cell's preference for curved contour stimuli.

The response histograms in Figure 1, $A$ and $B$, bottom, show the mean response + SEM to the best stimulus within each of the four grating subclasses and the ten contour subclasses denoted by the underlying icons. The small size of the error bars relative to the peak responses and to the range of response magnitudes indicates that neural responses were reasonably consistent in these cells (also see legend to Fig. 1). This suggests that these cells may convey significant information about shape differences across stimulus subclasses, an issue we address quantitatively below. Together, the foregoing observations show that individual V2 cells can explicitly encode information about a variety of shape characteristics, and that a given cell's responses to simple bars and gratings may not be predictive of the preferred stimulus or the overall response profile of the cell to a larger stimulus repertoire.

\section{Population analyses of shape selectivity}

We performed quantitative analyses to address the following questions about the shape selectivity of individual V2 neurons: (1) Is the cell's preferred stimulus a simple or a geometrically complex shape, and is the preference statistically significant? (2) Is the distribution of cells preferring each of the complex shape subclasses uniform or biased? (3) Do individual cells convey information about shape characteristics that are not attributable to stimulus orientation, size, or spatial frequency? (4) Do individual cells convey information about orientation, size, and/or spatial frequency within the preferred stimulus subclass? These analyses were performed separately for grating and contour stimuli, with the more striking results emerging from the analysis of contours.

In Figure $2 A$ each cell was assigned to one of the four grating subclasses (see Materials and Methods) based on its most effective grating stimulus. Most cells (110 of 180, 61\%) preferred one of the three subclasses of non-Cartesian grating, whereas 70 cells 


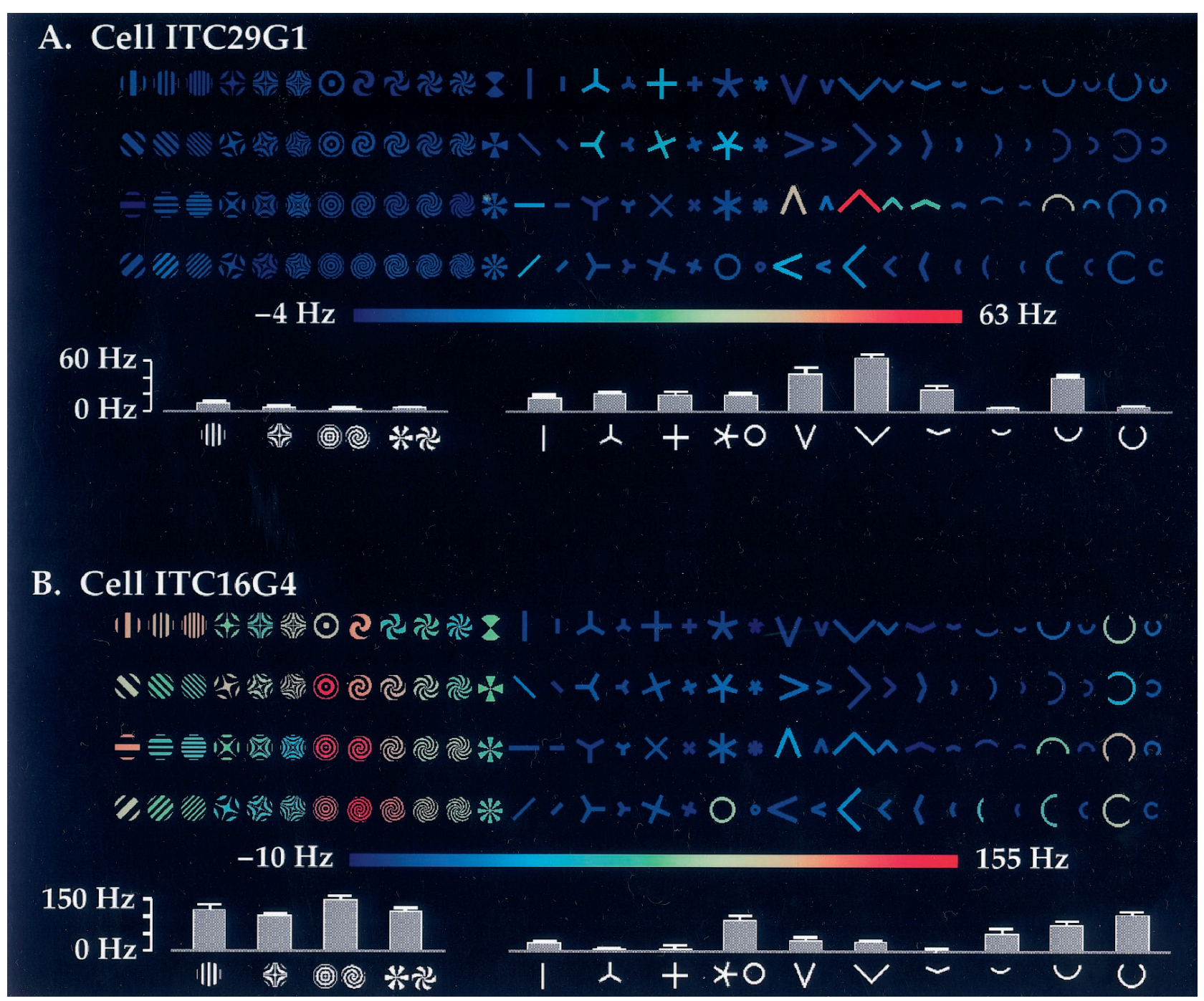

Figure 1. Responses of V2 cells to grating and contour stimuli. Color-coded mean responses of two individual cells $(A, B)$ to each of the 128 stimuli in the stimulus set are shown according to the linear color scale below each panel. In both panels, stimulus orientations were normalized so that the preferred orientation (as determined during the manual mapping of the receptive field) is shown as vertical. The bar plots at the bottom of each panel show the mean responses + SEM of the given cell to the most effective stimuli in each grating and contour stimulus subclass (see Materials and Methods) represented by icons underneath each bar. Such subclass peak responses from each V2 cell were used in the randomization analysis presented in Figures 2 and 3. In general, response variances were small compared with the corresponding response means for the exemplar cells as well as the V2 cell population at large. For the population as a whole, the mean SEM was 6.4 (or 14.3\% of the population mean of the subclass peak responses; data not shown). The response variance attributable to the spatial jittering of each stimulus within the receptive field was also low (data not shown). In a few cells that responded poorly to oriented bars used as mapping stimuli (e.g., $A$ ), the preferred orientation determined during the initial manual mapping differed from that determined from the recorded responses.

$(39 \%)$ responded best to sinusoidal gratings. Five of the 110 cells (5\%) responded significantly better to the most effective nonCartesian grating than to the most effective simple grating (onetailed $t$ test, $p<0.05$ after Bonferoni correction; see Materials and Methods), an incidence that is not larger than expected by chance. This is likely attributable to the fact that many V2 cells were broadly (although systematically) tuned to grating stimuli as a class (e.g., see Fig. $1 B$ ).

The corresponding analysis of the most effective contour stimuli is shown in Figure $2 B$. A large majority of the cells (152 of 180, $84 \%$ ) preferred a complex contour over the most effective bar stimuli. In 35 cells (19\%), this preference was statistically significant ( $p<0.05$, after Bonferoni correction). Nearly threequarters of the cells (112 of $152,74 \%)$ that preferred a complex contour over the most effective bar stimulus also preferred the complex contour over the most effective sinusoidal grating. One sixth of the cells (30 of 180,17\%) preferred acute angles over other contour stimuli. Both the overall distribution of cells preferring complex contours and the distribution of cells having a significant complex contour preference were nonrandom $(p<$ 0.005 and 0.05 , respectively), as determined by randomization analysis (see Materials and Methods). Only 28 of the $180(16 \%)$ cells responded best to a bar stimulus, and only two cells $(1 \%)$ responded significantly better to the most effective bar stimulus than they did to the most effective complex contour stimulus.

\section{Selectivity for various shape characteristics}

Whether cells such as those in Figure 1 carry significant amounts of information about various complex shape characteristics depends on the extent to which the variable responses to different 
Figure 2. Distribution of peak responses. $A$, Bar histogram of $180 \mathrm{~V} 2$ cells, each classified according to whether its most effective stimulus was sinusoidal, hyperbolic, concentric-like, or radial-like (see Materials and Methods). Icons below each bar represent the corresponding stimulus shapes. Cells indicated in black showed statistically significant preference for that subclass ( $p<0.05$ after Bonferoni correction; see Results). $B$, Similar analysis of the same 180 cells for their preference for contour subclasses. Of the 20 cells that preferred either a star or a circle stimulus, 11 cells (including all 4 cells with a statistically significant preference) preferred circles. The exemplar cells shown in Figure 1, $A$ and $B$ (denoted by a square and triangle, respectively), significantly preferred a right angle and a three-quarter arc, respectively, as shown. A sinusoidal and a concentric grating, respectively, were the most effective grating stimuli of the two cells, but not at a statistically significant level ( $p>0.05$ after Bonferoni correction). The stimulus subclasses that were preferred by a greater or a smaller number of cells than expected by chance (twotailed binomial probability test) are marked by single asterisks $(p<0.05)$ or double asterisks $(p<0.01)$.
A.

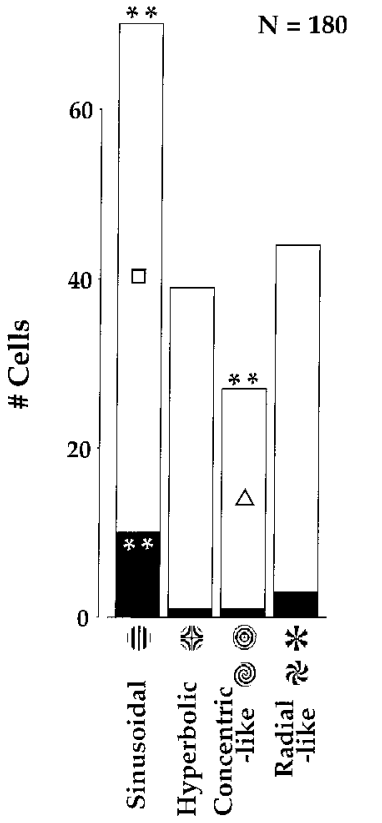

Grating Shape Classes
B.

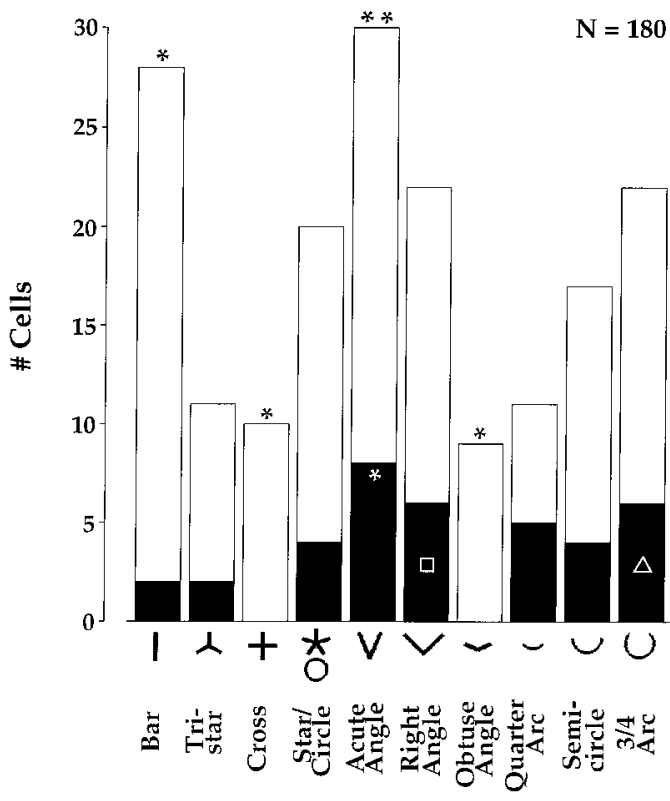

Contour Shape Classes stimuli exceed the trial-by-trial variability of responses to identical stimuli. In Figure 3, we address this issue for the peak responses within each complex stimulus subclass (Fig. 3A, B, horizontal axes) and for all of the stimuli within the complex shape subclass that elicited the largest peak response (Fig. $3 A, B$, vertical axes).

To determine the modulation of a given cell's responses by different subclasses of non-Cartesian gratings, we first calculated the variance of the cell's responses to its most effective hyperbolic, concentric-like and polar-like gratings. We then used the randomization analysis to determine the extent to which this variance differed from random. We calculated a complex grating shape selectivity index (CGSS), defined as the ratio between the actual variance of the three peak responses and the averaged randomized variance. We also calculated a within-preferred subclass selectivity index for gratings $\left(\mathrm{WPS}_{\mathrm{G}}\right)$, defined as the ratio between the actual and the randomized variances of the cell's responses to the 12 stimuli in the subclass containing the cell's most effective non-Cartesian grating stimulus. These two indices together measure the modulation of the cell's responses by one or more shape characteristics.

Figure $3 A$ shows the modulation of $\mathrm{V} 2$ cell responses by grating stimuli. For approximately one-tenth of the cells (19 of 180,11\%), the variance of the responses to complex gratings was significantly higher than that expected from random $(p<0.05)$, indicating that these cells were able to discriminate among subclasses of non-Cartesian grating. These 19 cells had a mean CGSS value of 4.7, reflecting a response variance 4.7-fold larger on average than that expected from random. For more than one-third of the cells (67 of $180,37 \%$ ), the response variance within the subclass containing the preferred complex grating was significantly higher than that expected from random $(p<0.05)$, with an average $\mathrm{WPS}_{\mathrm{G}}$ value of 3.1 for this subgroup.

To perform the corresponding analysis for complex contour stimuli (Fig. 3B), we calculated a complex contour shape selectivity index (CCSS), defined as the ratio between the actual variance of the cell's responses to the preferred stimuli from each of the nine complex contour stimulus subclasses and the average randomized variance. We also calculated a within-preferred subclass selectivity index for contours $\left(\mathrm{WPS}_{\mathrm{C}}\right)$, defined as the ratio between the actual and the randomized variances of the cell's responses to the eight stimuli in the subclass containing the cell's most effective complex contour stimulus. For approximately onethird of the cells (62 of 180, 34\%), the variance of the responses to complex contours was significantly higher than random $(p<$ $0.05)$. These 62 cells had a mean CCSS value of 3.5, reflecting a response variance 3.5 -fold greater on average than that expected from random. V2 cells also showed strong response modulation within the subclass containing their most effective complex contour stimulus. For about four-fifths of the cells (147 of 180, 82\%), this response variance was significantly higher than that expected from random $(p<0.05)$, with an average $\mathrm{WPS}_{\mathrm{C}}$ value of 4.4. For the contour responses, the indices for selectivity across stimulus subclasses (CCSS) and within the preferred subclass $\left(\mathrm{WPS}_{\mathrm{C}}\right)$ were strongly correlated with each other $(r=0.81 ; p<0.01)$. Of the 52 cells in which both indices exceeded 2.0, cells with an angle or an arc as the most effective stimulus were the most prevalent (19 and 15, respectively), and those preferring an intersection or a bar were least prevalent (10 and 8, respectively).

The above results indicate the responses of many V2 cells were modulated by complex shape characteristics and to an even greater extent by the orientation, size, and/or spatial frequency of the preferred shape. Taken together with the results of the peak response analysis, these results indicate that many V2 cells carry substantially detailed and potentially useful information about complex forms.

\section{DISCUSSION}

\section{Role of $\mathbf{V} 2$ in form processing}

This study provides the most detailed and systematic characterization to date of the responsiveness and selectivity of neurons in macaque area V2 to complex shapes. Our results suggest a broad 

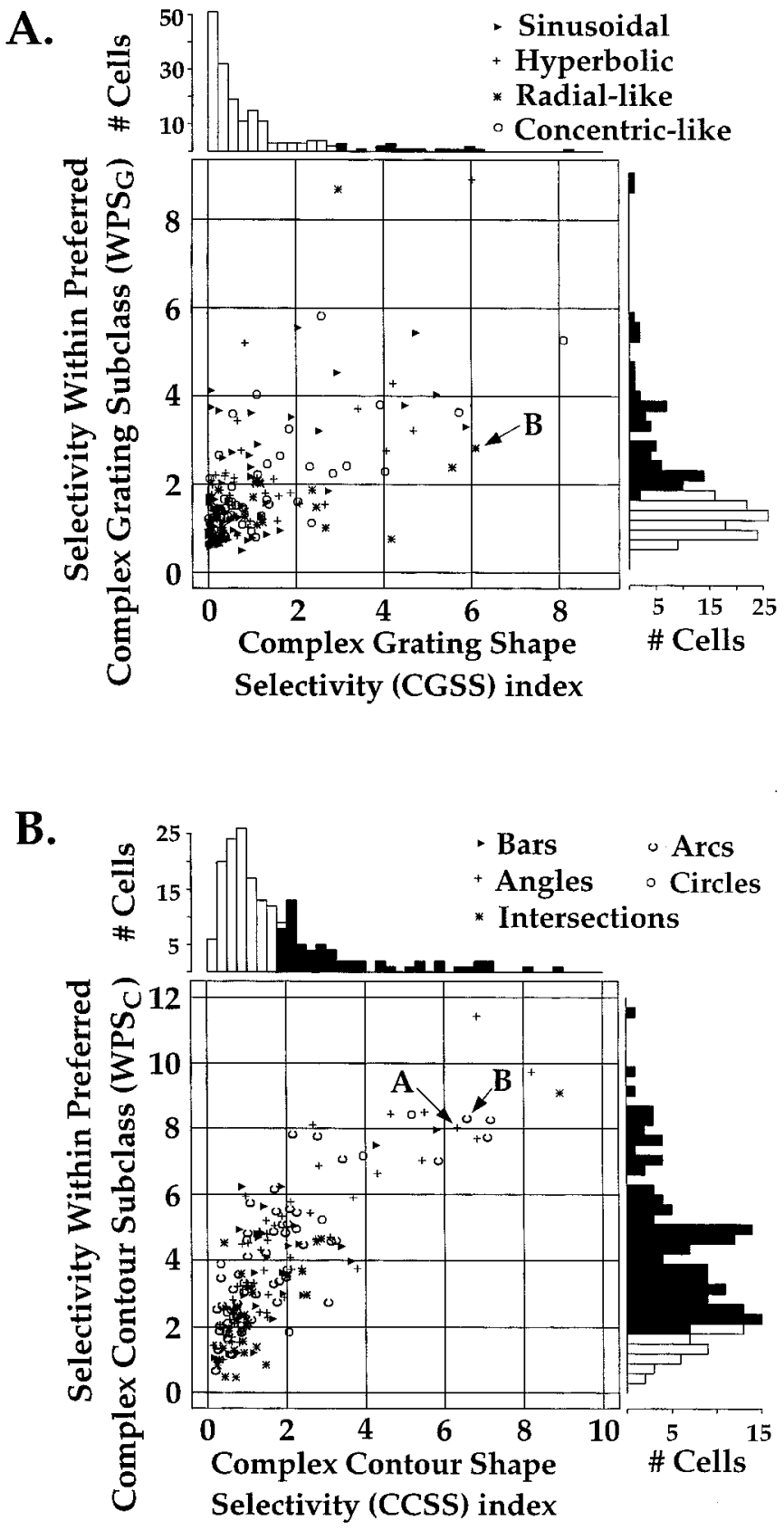

Figure 3. Modulation of V2 cell responses by complex shapes. Each symbol represents a single cell; the shape of the symbol represents the cell's most effective grating $(A)$ or contour $(B)$ stimulus according to the key provided. In $B$, cells preferring tristars, crosses, and stars are represented collectively as intersections. Cells preferring angles, arcs, and circles are also grouped into a class each. $A$, Response modulation by non-Cartesian gratings. A CGSS index and a WPS $\mathrm{W}_{\mathrm{G}}$ index were calculated for each cell (see Results) and plotted along the $x$ - and $y$-axes, respectively. The cell shown in Figure $1 B$ is denoted by an arrow; the cell shown in Figure $1 A$ was located within the cluster of cells near the origin. The distribution of index values on either axis is shown in histogram form on the corresponding axis; the cells shown in black represent the cells for which the response variance among stimulus types was significantly different from random ( $p<0.05$; see Materials and Methods). $B$, Corresponding analysis of complex contour responses of the $180 \mathrm{~V} 2$ cells. The

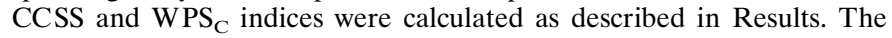
cells shown in Figure 1, $A$ and $B$, are denoted by arrows. role for area $\mathrm{V} 2$ in form analysis, wherein V2 cells collectively encode information about many complex shape characteristics. Together with the known responsiveness of V2 neurons to subjective contours (Peterhans and von der Heydt, 1993), these findings indicate that the role of V2 in form processing cannot be fully explored using conventional bar and sinusoidal stimuli alone. It is also evident that complex stimuli can be more effective than conventional bars and sinusoids for the purposes of driving V2 cells, e.g., as mapping stimuli.

Our results also suggest specific ways in which V2 cells may encode complex form information. For instance, the selectivity of V2 cells for complex contour stimuli suggests that V2 cells encode information about various aspects of object and surface boundaries. Many V2 cells are selective for additional contour characteristics, such as the sign of curvature and/or the polarity of angles (e.g., " $<$ " vs " > "). The higher incidence of cells selective for acute angles versus obtuse angles (e.g., Fig. 2B) is especially noteworthy. Acute angles often occur at object corners and at the intersection of occluding contours, both of which are perceptually salient.

Selectivity for non-Cartesian gratings has been previously reported in area V4 (Gallant et al., 1996), but the present study is the first to demonstrate that such cells exist in V2, although not in large numbers. The responsiveness of most V2 cells to grating patterns and the selectivity of some for non-Cartesian gratings allows these cells to encode information about the textural composition and textural gradients within object interiors as well as in background patterns (Gallant et al., 1996).

Modeling studies suggest that distinct mechanisms may underlie the processing of boundaries and surfaces (Grossberg, 1987) (also see Zucker, 1985). Insofar as the selectivity for grating patterns and contours might reflect the analyses of surface characteristics and boundaries, respectively, our results are relevant to such hypotheses. However, further studies are needed to reveal the degree to which surface and boundary processing are physiologically distinct at the level of V2.

\section{Origins of $\mathbf{V} \mathbf{2}$ receptive field properties}

The selectivity of V2 cells for complex contours and gratings raises the intriguing question of how these receptive field properties arise. One possibility is that these properties arise de novo in V2 by an appropriate pattern of ascending inputs from cells in $\mathrm{V} 1$ selective for low-order form information such as orientation and/or spatial frequency (see Riesenhuber and Poggio, 1999). For instance, selectivity for a right angle might arise as a result of inputs from two subsets of V1 cells, each selective for one of the two orthogonal orientations, much as originally proposed for "higher order hypercomplex" cells in cat area 19 by Hubel and Wiesel (1965). Selectivity for complex shapes in V2 may also arise from lateral inputs from V2 cells selective for simple stimuli and/or descending inputs from similar cells in higher visual areas such as V4 (Gallant et al., 1996). Finally, the properties of V2 cells might reflect complex shape preferences of V1 cells themselves. This could arise from nonclassical surround interactions (Knierim and Van Essen, 1992; Lamme, 1995; Das and Gilbert, 1999) or from selectivity within the classical receptive field to complex shapes (Versavel et al., 1990; Hegdé and Van Essen, 1999).

\section{REFERENCES}

Burkhalter A, Van Essen DC (1986) Processing of color, form and disparity in visual areas VP and V2 of ventral extrastriate cortex in the macaque monkey. J Neurosci 6:2327-2351. 
Connor CE, Preddie DC, Gallant JL, Van Essen DC (1997) Spatial attention effects in macaque area V4. J Neurosci 17:3201-3214.

Das A, Gilbert CD (1999) Topography of contextual modulations mediated by short range interactions in primary visual cortex. Nature 399:655-661.

DeValois RL, DeValois KK (1988) Spatial vision. New York: Oxford UP.

Dobbins A, Zucker SW, Cynader MS (1987) Endstopped neurons in the visual cortex as a substrate for calculating curvature. Nature 329:438-441.

Gallant JL, Connor CE, Rakshit S, Lewis JW, Van Essen DC (1996) Neural responses to polar, hyperbolic, and Cartesian gratings in area V4 of the macaque monkey. J Neurophysiol 76:2718-2739.

Grossberg S (1987) Cortical dynamics of three-dimensional form, color, and brightness perception. Percept Psychophys 41:87-158.

Hegdé J, Van Essen DC (1997a) Selectivity for complex contour stimuli in visual area V2. Invest Ophthalmol Vis Sci 38:S969.

Hegdé J, Van Essen DC (1997b) Selectivity for non-Cartesian and conventional gratings in visual area V2. Soc Neurosci Abstr 23:1395.

Hegdé J, Van Essen DC (1999) Selectivity for complex shapes in primate visual area V1. Soc Neurosci Abstr 25:1548.

Hubel DH, Wiesel TN (1965) Receptive fields and functional architecture in two non-striate visual areas (18 and 19) of the cat. J Neurophysiol 28:229-289.
Huberty CJ, Morris JD (1989) Multivariate analysis versus multiple univariate analyses. Psychol Bull 114:145-161.

Kobatake E, Tanaka K (1994) Neuronal selectivities to complex object features in the ventral visual pathway of the macaque cerebral cortex. J Neurophysiol 71:856-867.

Lamme VAF (1995) The neurophysiology of figure-ground segregation in primary visual cortex. J Neurosci 15:1605-1615.

Manly BFJ (1991) Randomization and Monte Carlo methods in biology. New York: Chapman and Hall.

Pasupathy A, Connor CE (1999) Responses to contour features in macaque area V4. J Neurophysiol 82:2490-2502.

Peterhans E, von der Heydt R (1993) Functional organization of area V2 in the alert macaque. Eur J Neurosci 5:509-524.

Reid RC, Alonso JM (1996) The processing and encoding of information in the visual cortex. Curr Opin Neurobiol 6:475-480.

Riesenhuber M, Poggio T (1999) Hierarchical models of object recognition in cortex. Nat Neurosci 2:1019-1025.

Versavel M, Orban GA, Lagae L (1990) Responses of visual cortical neurons to curved stimuli and chevrons. Vision Res 30:235-248.

Zucker SW (1985) Early orientation selection: tangent fields and the dimensionality of their support. Comput Vision Graphics Image Processing 32:74-103. 